\title{
Evolution of Wolf-Rayet stars in binary systems: analysis of distributions of masses and orbital eccentricities
}

\author{
Anatol M. Cherepashchuk ${ }^{1}$ and Valentin G. Karetnikov ${ }^{2}$ \\ ${ }^{1}$ Sternberg Astronomical Institute, \\ 13 Universitetskii Prospekt, Moscow 119992, Russia \\ ${ }^{2}$ Astronomical Observatory, Odessa University, \\ Odessa, 270029, Ukraine
}

Abstract. It is shown that up to $90 \%$ of the known WR+O binaries have been formed as a result of the mass transfer in $\mathrm{O}+\mathrm{O}$ binary systems.

To check Moffat's (1995) hypothesis concerning stellar wind evolution of O+O binaries, the distribution of stellar masses and eccentricities of binary orbits were investigated for different binary system types: WR+O close binaries, Main Sequence Detached (MSD) binaries, Early-type Contact (EC) binaries, and SemiDetached (SD) binaries.

$\mathrm{WR}+\mathrm{O}$ binary characteristics have been compared to corresponding characteristics of MSD, EC and SD-systems. The comparison allows us to conclude that up to $90 \%$ of the known WR+O binaries has been formed as a result of mass transfer in $\mathrm{O}+\mathrm{O}$ systems (Cherepashchuk \& Karetnikov 2003).

Since components of SD-systems with subgiant stars are shown to have a certain correlation as respect to their masses, the fact that anticorrelation is not revealed between $\mathrm{WR}$ and $\mathrm{O}$ masses in WR+O binaries can not be an argument against $\mathrm{WR}+\mathrm{O}$ formation as a result of mass exchange (Figure 1).

The spectroscopic transitional orbital period $P_{\mathrm{tr}}^{\mathrm{sp}}$ which corresponds to transition between nearly circular orbits $\left(e_{\mathrm{sp}}<0.1\right)$ and elliptical ones $\left(e_{\mathrm{sp}} \geq 0.1\right)$ is equal to $\sim 14 \mathrm{~d}$ for $\mathrm{WR}+\mathrm{O}$ systems and $\sim 2-3 \mathrm{~d}$ for $\mathrm{OB}+\mathrm{OB}$ systems (Figure 1).

Periods $P_{\mathrm{tr}}^{\mathrm{sp}}$ are strongly different for $\mathrm{WR}+\mathrm{O}$ and $\mathrm{OB}+\mathrm{OB}$ binary systems, which indicates that there is an extra mechanism making orbits circular in $\mathrm{WR}+\mathrm{O}$ binaries. This extra mechanism is additional to that which was actuated in $\mathrm{OB}+\mathrm{OB}$ binary systems at the MSD-stage (radiative suppression of dynamical tides) and is likely to be connected with filling its Roche Lobe by a primary O-type star and the mass transfer in original MSD O+O binaries.

Most of the SD-binaries which are known to contain subgiant stars had their orbits already circularized at the MSD-stages, before mass transfer processes began. Thus, mass transfer is unlikely to play a significant role in circularizing orbits for most SD-binaries.

Since the mass transfer in original $\mathrm{O}+\mathrm{O}$ binary systems does not last long, the number of orbital cycles in such systems in their initial stage of mass transfer is relatively small. The further mass transfer from a low-mass star to its massive companion, after the component masses have become equal, results in $\mathrm{WR}+\mathrm{O}$ 

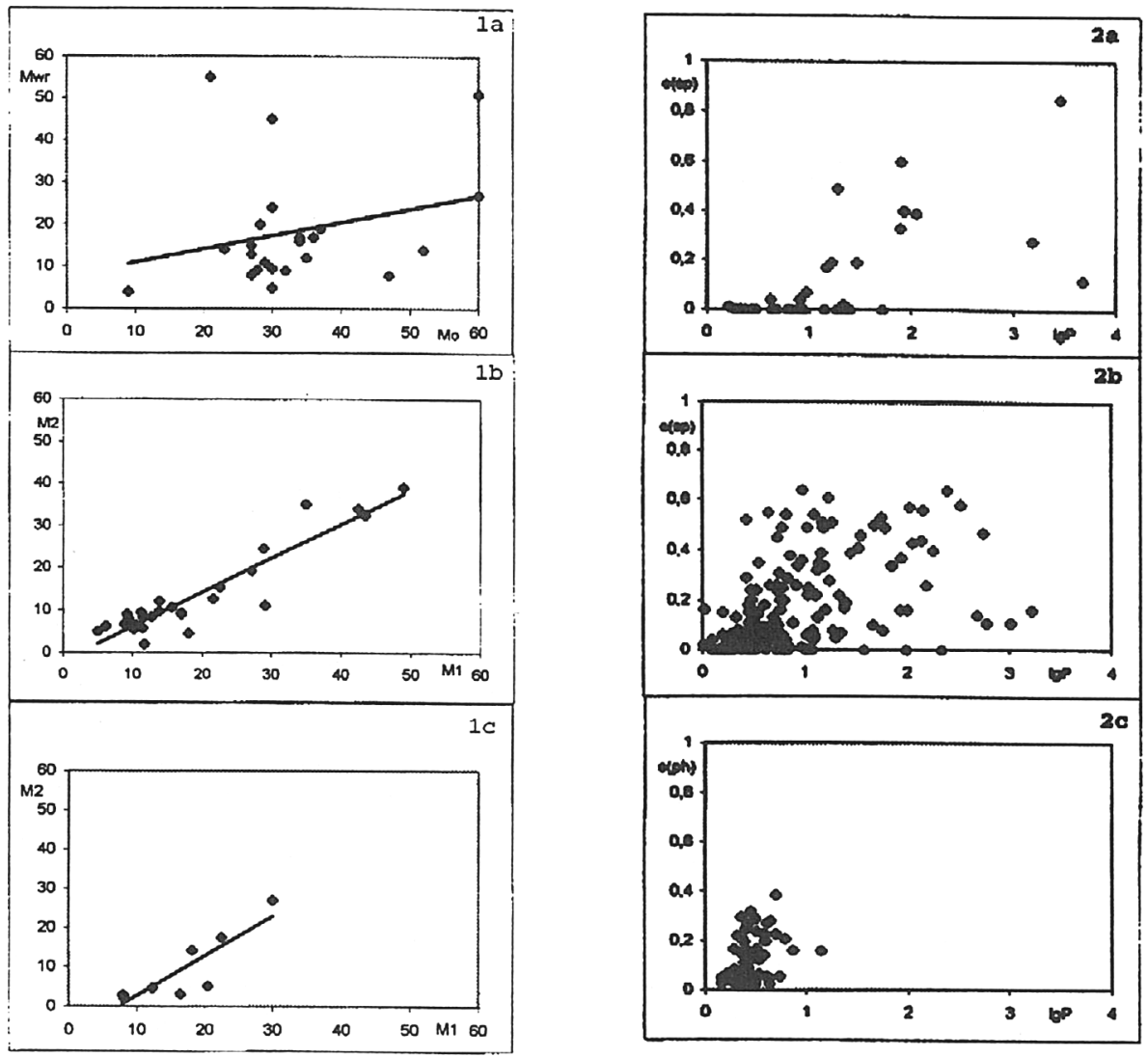

Figure 1. Left: Correlation between masses of components in WR+O (1a), EC (1b), and SD binaries (1c). Right: Eccentricities of the orbits vs. the logarithm of orbital periods in close binaries: WR+O (2a), OB+OB (2b, 2c).

binary formation and increase in the residual eccentricity of the orbit up to observable values. The orbital eccentricity may also increase due to variable radial mass loss in the form of stellar wind from the WR star, while circling in elliptical orbit in a WR+O binary.

So WR+O binary systems with long orbital periods having considerable orbital eccentricities can not argue unambiguously against mass exchange in original $\mathrm{O}+\mathrm{O}$ binaries.

\section{References}

Cherepashchuk, A.M., Karetnikov, V.G. 2003, Astron. Reports 47, 38

Moffat, A.F.J. 1995, in: K.A. van der Hucht \& P.M. Williams (eds.), Wolf-Rayet Stars: Binaries, Colliding Winds, Evolution, Proc. IAU Symp. No. 163 (Dordrecht: Kluwer), p. 213 